\title{
Searching for an Optimal Reference System for On-Line Signature Verification Based on (x, y) Alignment
}

\author{
Juan J. Igarza, Lorea Gómez, Inma Hernáez, and Iñaki Goirizelaia \\ Department of Electronics and Telecommunications \\ University of the Basque Country \\ Alameda Urquijo s/n, 48013 Bibao, Spain. \\ \{jigarza, lorea, inma, igoirizelaia\}@bips.bi.ehu.es
}

\begin{abstract}
The handwritten signature is the expression of the will and consent in daily operations such as banking transactions, access control, contracts, etc. However, since signing is a behavioral feature it is not invariant; we do not always sign at the same speed, in the same position or at the same orientation. In order to reduce the errors caused in verification by these differences between original signatures we have introduced a new concept of reference system for the $(\mathrm{x}, \mathrm{y})$ coordinates from experimental results. The basis of this concept lies on using global references (centre of mass and principal axes of inertia) instead of local references (initial point and initial angle) for a recognition system based on local parameters. The system is based on the hypothesis that signing is a feedback process, in which humans react to our own signature while writing it following patterns stored in our brain.
\end{abstract}

\section{Introduction}

Traditionally, the dynamic handwritten signature verification process by means of Hidden Markov Models (HMM) consists on the following stages: digitization of the signature, processing, features extraction, verification and finally decision.

From the graphics tablet the $(\mathrm{x}, \mathrm{y})$ coordinates that describe the movement of the signer's hand along the paper are obtained. These coordinates show the position of the pen every $10 \mathrm{~ms}$, obtaining a sequence of samples. Due to signature variability, the signal extracted from the tablet can differ for the same signing person, depending on the speed of writing, the size of the signature, its position and orientation.

The causes of this variability can be divided into two groups. On one hand the number of samples of the sequence depends on the length of the signature and on its size [1], since the writer does not always last the same in signing and the physical space provided to sign may vary. On the other hand, the coordinates must be processed to avoid variability due to the position and orientation of the signature since from the corrected $(\mathrm{x}, \mathrm{y})$ coordinates several characteristics will be extracted, such as speed or acceleration, to be used in the verification process.

In fact, the signature may be made in different positions of the tablet at different instants so coordinates should be referenced to an alignment point. Moreover, due to 
rotations in signer's hand or in the tablet the orientation of the signature is variable, so the angle of the reference axes is also needed.

In this sense, some authors [2] propose the use of the first sample of the signature as a reference in order to reduce position variability and some others suggest the initial angle [3] to avoid rotation variability.

This paper discusses the variability of the $(x, y)$ coordinates and proposes a new method for processing the coordinates by means of an alignment based on global references. Two different aspects are researched in more detail: the use of the center of mass or centroid of ink as the center of the reference system in the alignment and the use of the principal axes of inertia to find the axes that best align the signatures.

\section{Reference Point for (x, y) Coordinates Alignment}

As stated, the signatures must be aligned for the enrollment and verification processes to make the $(\mathrm{x}, \mathrm{y})$ coordinates independent of the position of the tablet. We must find the best reference point in order to get the best alignment between the signatures.

a) Alignment using the initial sample of the signature as a reference When we use the first point of the signature $\left(\mathrm{x}_{0}, \mathrm{y}_{0}\right)$ as the center of the $(\mathrm{x}, \mathrm{y})$ coordinates reference system the alignment is carried out as follows:

$$
x^{\prime}=x-x_{0} \quad y^{\prime}=y-y_{0}
$$

b) Alignment using the center of mass as a reference

The $(\mathrm{x}, \mathrm{y})$ coordinates of the centroid of ink or centre of mass of the signature $(\mathrm{N}-$ particle system) are:

$$
x_{c m}=\frac{1}{N} \sum x \quad y_{c m}=\frac{1}{N} \sum y
$$

In this case, the alignment is carried out as follows:

$$
x^{\prime}=x-x_{c m} \quad y^{\prime}=y-y_{c m}
$$

The center of mass is calculated applying the Breshingham algorithm to every two consecutive samples of the signature. This leads to involve in the computation not only the pen-down samples but also intermediate points that are calculated by means of this segment approach algorithm.

Figure 1 shows the signatures of two users and the result of superimposing ten signatures referred to the coordinates system centered in the initial point (Fig. 1b) or the center of mass (Fig. 1c). As can be seen in the figures, the global dispersion is lower when the signatures are referred to the center of mass. Namely, we get a better alignment of the signatures with little deviation in $\mathrm{x}$ and $\mathrm{y}$.

\section{Reference Angle for the (x, y) Coordinates Alignment}

The variability in the orientation of the signature produces noise in the verification. As stated before, the rotation may be caused by the writer's hand or by the position of the graphics tablet. This is why once the center of the new reference system is 
defined; we should find a suitable reference angle to rotate the system axes. Once calculated, the coordinates will be rotated by means of a rotation matrix as follows:

$$
x^{\prime}=x \cos \theta-y \operatorname{sen} \theta, \quad y^{\prime}=x \operatorname{sen} \theta-y \cos \theta
$$

where $\mathrm{x}$ and $\mathrm{y}$ are the original coordinates, $x^{\prime}$ and $y^{\prime}$ the new ones, and $\theta$ the reference angle.

a) Alignment using the initial angle as reference

In this case the angle of reference is calculated as follows:

$$
\theta_{0}=\operatorname{arctg}\left(\frac{y_{1}-y_{0}}{x_{1}-x_{0}}\right)
$$

From experimental results in previous studies [4] we know that this is a noisy angle. Figure 2 shows three signatures of the same user rotated by the initial angle and superimposed (the left side of the figure shows a zoom of the origin). It can be seen that the rotation by the initial angle does not get the correct alignment. Quite the opposite, it introduces noise since the initial angle might be random.

b) Alignment using the angle of minimum inertia as reference

The principal axes of inertia of a rigid body are a set of axes passing through the object's center of mass and relate to the object's mass distribution such that angular momentum imparted about a principal axis is not transferred to any other axis. The symmetry axis of a pencil is one example of a principal axis. An asymmetric twodimensional signature has two mutually perpendicular principal axes, which can always be calculated unambiguously. One of them is known as the minimum moment of inertia.

Fig. 1a

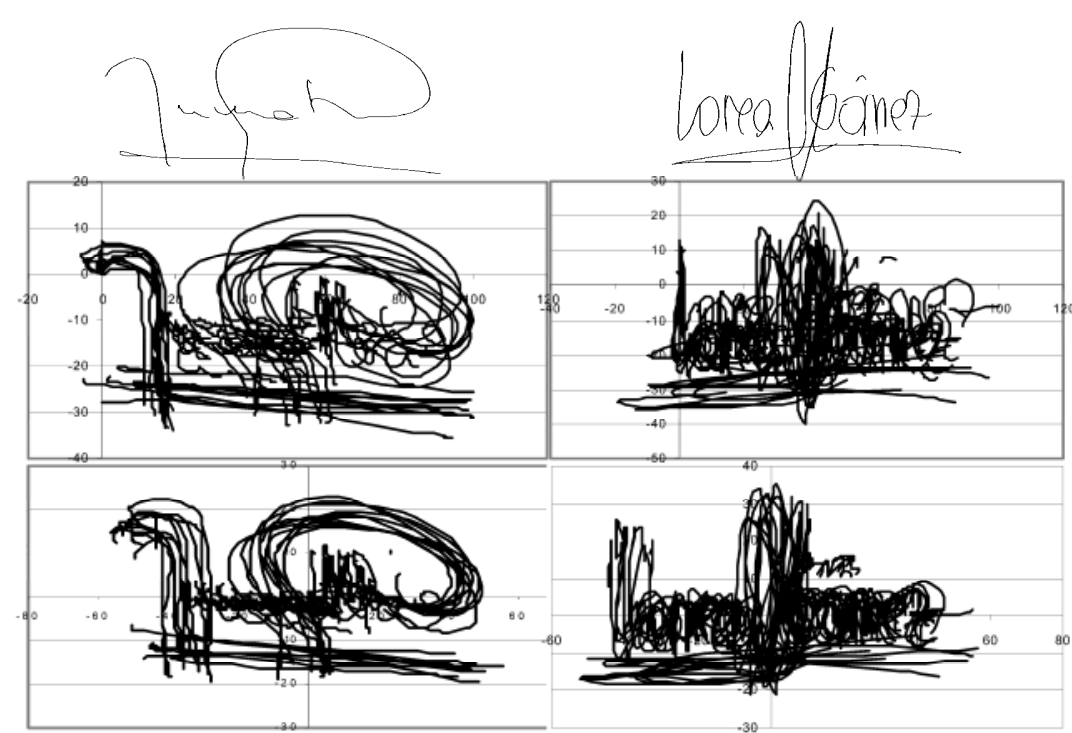

Fig. 1. 1a two signatures of two users. 1b. Graphical superimposition of ten signatures referred to the coordinates system centered in the initial point. 1c: Graphical superimposition of ten signatures referred to the coordinates system centered in the mass center 


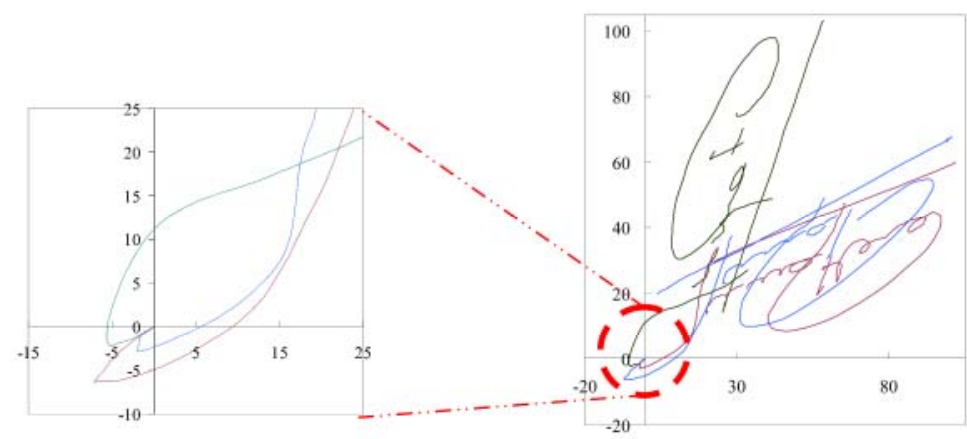

Fig. 2. Three signatures of the same user rotated by the initial angle and superimposed. The left side of the figure shows a zoom of the origin

The angle of minimum inertia gives an indication of the orientation of the signature, and there is a direct relationship between the angle of inertia and the writing trend.

To avoid variability in orientation in the process of matching the acquired data with the reference data we should rotate the $(x, y)$ coordinates of every signature by an angle that equals the angle of minimum moment of inertia but in the opposite direction. Namely, we align all the signatures so as to make the verification independent of the writing direction and eliminate noise.

The angle of minimum inertia $\theta$ is obtained using the formula below:

$$
\theta=\frac{1}{2} \arctan \left[\frac{2 I_{x y c m}}{I_{y c m}-I_{x c m}}\right]
$$

where $\mathrm{I}_{\mathrm{xcm}}, \mathrm{I}_{\mathrm{ycm}}$ and $\mathrm{I}_{\mathrm{xycm}}$, are the moments of inertia referred to the axes parallel to the origin of coordinates and crossing the center of mass. They are calculated using the following procedure:

I. First, the moments of inertia referred to the origin of $(x, y)$ coordinates and the crossed moment of inertia are calculated as follows:

$$
I_{x 0}=\sum y^{2}, I_{y 0}=\sum x^{2}, I_{x y 0}=\sum x y
$$

II. Secondly, these moments must be referenced to the previously calculated center of mass. This problem is solved by means of the Steiner theorem:

$$
I_{o}=I_{c m}+d^{2} N
$$

where,

- Icm is the moment of inertia referred to an axis that crosses the center of mass, $I_{o}$ is the moment referred to an axis parallel to the previous one and $d$ is the distance between the two parallel axes.

- $\quad N$ is the number of pen down samples that form the signature and the intermediate points calculated by the Breshingam algorithm.

In this way, the moments of inertia referred to the center of mass can be calculated using:

$$
I_{x c m}=I_{x 0}-N y_{c m}{ }^{2}, I_{y c m}=I_{y 0}-N x_{c m}{ }^{2}, I_{x y c m}=I_{x y 0}-N x_{c m} y_{c m}
$$




\section{Results}

We have performed the evaluation of the proposed references over a 100 user's database [5]. This database contains 25 original signatures from each user, and 25 skilled forgeries of each user. The verification process is carried out by means of the Hidden Markov Models. We have chosen a Left to Right topology with a fixed number of states (six) so as to obtain satisfactory results in generating the model and in the verification process.

The experiments involve the use of different sets of parameters:

a) Training of the HMM's using as a parameter set only ( $x, y$ ) coordinates referred to the center of mass and to the initial sample.

b) Training of the HMMs using only the $(x, y)$ rotated coordinates referred to the center of mass and not rotated but referred to the center of mass.

c) Training of the HMMs using all the available parameters (rotated coordinates and seven more parameters: pressure, azimuth, acceleration, inclination, acceleration in $\mathrm{x}$ axis, linear and angular speed).

Carrying out the verification using only the $(\mathrm{x}, \mathrm{y})$ coordinates referred to the center of mass and the initial sample we get the EER (Equal Error Rate) values shown in Table 1. As can be seen, an important improvement is achieved when using the center of gravity as reference.

Training the system with the rotated coordinates has given different results depending on the kind of signature:

Case 1 Small variation in the EER

For the majority of the users $(72 \%)$ the EER has no varied (+-4\% deviation using the rotated or non-rotated coordinates in the verification).

Table 1: Comparison of the EER for the verification using only $(x, y)$ coordinates referenced to the center of mass $(\mathrm{cm})$ or the initial point (ip)

\begin{tabular}{cccc}
\hline & EER cm (\%) & EER ip (\%) & $\Delta$ EER (\%) \\
\hline Mean value & $24.4 \%$ & $42.16 \%$ & $-17.76 \%$ \\
\hline
\end{tabular}

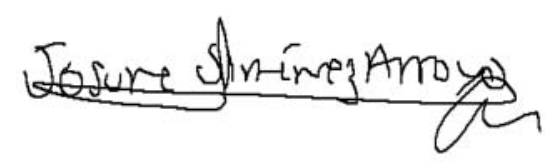

Fig. 3. As an example of EER improvement by means of coordinates rotation we show one signature of a user where the angle of minimum inertia varies in the range $\left[-2.72^{\circ}, 1.62^{\circ}\right]$ among the 25 original signatures in the database 

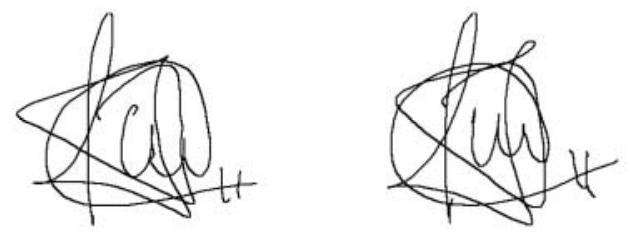

Fig. 4. Two signatures of the same user for which the deviation of the minimum angle of inertia is $77.11^{\circ}\left(\left[-38.77^{\circ}, 38.34^{\circ}\right]\right)$ among the 25 signatures of the database

Case 2 Improvement in the EER

The rotation of the coordinates leads to a remarkable improvement of the EER for those signatures that have a nearly constant orientation (that is, the angle of minimum inertia presents little deviation). The improvement is especially important for those signatures with a horizontal trend, since for these ones the user has a precise reference and he/she is able to keep the same orientation at every time he/she signs. Figure 3 shows one example of this case.

Case 3 Worsening of the EER

There are some signatures for which the instability in writing provokes a wide deviation in the angle of minimum inertia. For those signatures, if we use rotated coordinates in the verification the results are worse than when we use non-rotated coordinates. Figure 4 shows an example of this case. The signatures of this user show a deviation of $77.11^{\circ}\left(\left[-38.77^{\circ}, 38.34^{\circ}\right]\right)$ among his 25 signatures of the database. This is due to the instability of the signature's shape.

The instability found in the signature can be related with the EER found in the verification process using only the $(\mathrm{x}, \mathrm{y})$ coordinates. Considering that a user presents a stable performance if he or she gets an EER less than or equal $20 \%$, we find that approximately half of the signers $(52 \%)$ are stable in our database. Using only this set of stable signers, the EER does not show an improvement, as shown in table 2.

Table 2. Comparison of the EER for the verification using only $(x, y)$ coordinates rotated the initial angle and the angle of minimum inertia

\begin{tabular}{ccc}
\hline & $\begin{array}{c}\text { EER with } \\
\text { rotated }(x, y)\end{array}$ & $\begin{array}{c}\text { EER with non- } \\
\text { rotated }(\mathbf{x}, \mathbf{y})\end{array}$ \\
\hline Mean value & $14.3 \%$ & $14.7 \%$ \\
\hline
\end{tabular}

Table 3. Final results of the project using 9 local parameters of the signatures using global references: $(x, y)$ coordinates referred to the canter of mass and rotated the minimum angle of inertia, pressure, azimuth, acceleration in $\mathrm{x}$ axis, linear and angular speed

\begin{tabular}{ccc}
\hline Mean EER & Mean FAR & Mean FRR \\
\hline $4.66 \%$ & $0 \%$ & $9.64 \%$ \\
\hline
\end{tabular}

Nevertheless, a worsening in the EER of the system trained only with the rotated coordinates does not imply a loss of effectiveness in the verification as long as for those kind of signatures the other parameters are used, and the verification success 
lies on those parameters. We trained the system with all the available parameters (coordinates referred to the center of gravity and rotated to the angle of minimum inertia, pressure, azimuth, acceleration in the $\mathrm{x}$ axis, and linear and angular speed).

\section{Conclusions}

As stated herein in spite of the fact that the way of signing is a behavioral feature, it presents certain variability. In fact, at first the signer places the hand on the tablet randomly; however, as long as he writes the signature becomes more stable in size, shape, speed... In other words, signing is a feedback process. This feedback is mainly visual, since if we try to sign with our eyes closed or with an inkless pen will make an amorphous signature.

In order to profit from this feedback, we propose the signature alignment based on global references. We have evidenced that using the center of gravity as the origin of the reference system instead of the initial sample improves the mean EER in $17.76 \%$. Furthermore, using as reference the axes obtained by rotating the main axes a quantity equal to the angle of minimum inertia an added improvement in the EER of $0.4 \%$ is obtained.

\section{References}

[1] V. S. Nalwa, "Automatic on-line signature verification", Proceedings of the IEEE vol 85 No 2, Feb 1997 pp. 215-239.

[2] Javier Ortega-Garcia, Joaquin Gonzalez-Rodriguez, Danilo Simon-Zorita y Santiago Cruz-Llanas, "From Biometrics Technology to Applications Regarding Face, Voice, Signature and Fingerprint Recognition Systems". "Biometric Solutions for Authentication in an e-World" Chapter 12. Ed: Kluwer Academic Publishers, 2002 pp. 312-319.

[3] L. Yang and B.K. Widjaja and R. Prasad, "Application of Hidden Markov Models for signature verification", Pattern Recognition, Vol 28, No 2, 1995.

[4] Juan J. Igarza, Iñaki Goirizelaia, Koldo Espinosa, Inmaculada Hernáez, Raúl Méndez, Jon Sánchez. "On-line Handwritten Signature Verification Using Hidden Markov Models" Progress in Pattern Recognition, Speech and Image Analysis. LNCS Vol. 2905 Springer November, 2003 pp. 391-399.

[5] J. Ortega, J. Fierrez, D. Simon, J. Gonzalez, I. Hernaez, J.J.Igarza, C. Vivaracho, D. Escudero, Q.I. Moro "MCYT baseline corpus: a bimodal biometric database", IEE Proceedings Vision, Image and Signal Processing. Vol. 150(6) December, 2003 pp 395 401. 